\title{
Lipoma gigante bilobular en la mano: Caso clínico y revisión de la literatura
}

\author{
F. Panizo Mota ${ }^{(1)}$, H. Fahandezh-Saddi Díaz ${ }^{(2)}$, Á. López Hualda ${ }^{(1)}$, \\ R. M. Egea Gámez ${ }^{(1)}$ H. Valencia García ${ }^{(2)}$ \\ Hospital Universitario Fundación Alcorcón. Unidad de Mano y Miembro Superior \\ (1) Médico Interno Residente de Cirugía Ortopédica y TRaumatología \\ (2) UnIDAD DE Cirugía de la MANO Y ExTREMIDAd SUPERIOR
}

\section{Correspondencia:}

Dr. Fernando Panizo Mota

Hospital Universitario Fundación Alcorcón

Servicio de Cirugía ortopédica y traumatología

C/ Budapest, $n^{\circ} 1$

28922 Alcorcón (Madrid)

Teléfono: 916219400

e-mail: fer.panizo@hotmail.com

\begin{abstract}
Los lipomas en la mano son infrecuentes y pueden asociar clínica compresiva de nervio mediano. Se denominan gigantes cuando superan $\operatorname{los} 5 \mathrm{~cm}$; en estos casos se debe hacer el diagnóstico diferencial con el liposarcoma. Describimos a continuación el caso de una paciente que presentó un lipoma gigante en la región palmar de la mano.
\end{abstract}

Palabras clave: mano, tumor, lipoma gigante
Lipomas on the hands are uncommon, and could associate with compression on the median nerve. When these are bigger than $5 \mathrm{~cm}$, are called giant lipomas, and must be distinguish from liposarcoma. Next, we'll describe a patient's case who presented a giant palmar lipoma.

Key words: hand, tumor, giant lipoma

Rev. Iberam. Cir. Mano - Vol. 39• Núm. 1 • Mayo 2011 (70-74)

\section{INTRODUCCIÓN}

T os lipomas son una proliferación benigna de adipocitos, con bajo riesgo de degeneración a liposarcomas. Un rápido crecimiento, dolor, gran tamaño, presencia de invasión local hacia otras estructuras locales en la resonancia magnética $(\mathrm{RM})$, pueden sugerir una enfermedad maligna. Los lipomas ocasionalmente son de gran tamaño, con gran ocupación y desplazamiento de estructuras vasculonerviosas. Se denomina lipoma gigante cuando el tumor es mayor de $5 \mathrm{~cm}^{1,2}$, como el caso que presentamos.

Se trata de una tumoración frecuente en cualquier lugar del organismo, aunque son raros en la palma y dedos de la mano, suponiendo esta última localización aproximadamente un 5\% del total de los lipomas del organismo. Esta rareza se confirma en numerosas series de casos y revisiones de la literatura ${ }^{3-5}$.

Presentamos el caso clínico de una mujer que presentaba un lipoma gigante en la región palmar que fue intervenida, dado el rápido crecimiento de la tumoración.

\section{CASO CLÍNICO}

Mujer de 59 años de edad, con una tumoración en la región palmar en la mano izquier- 


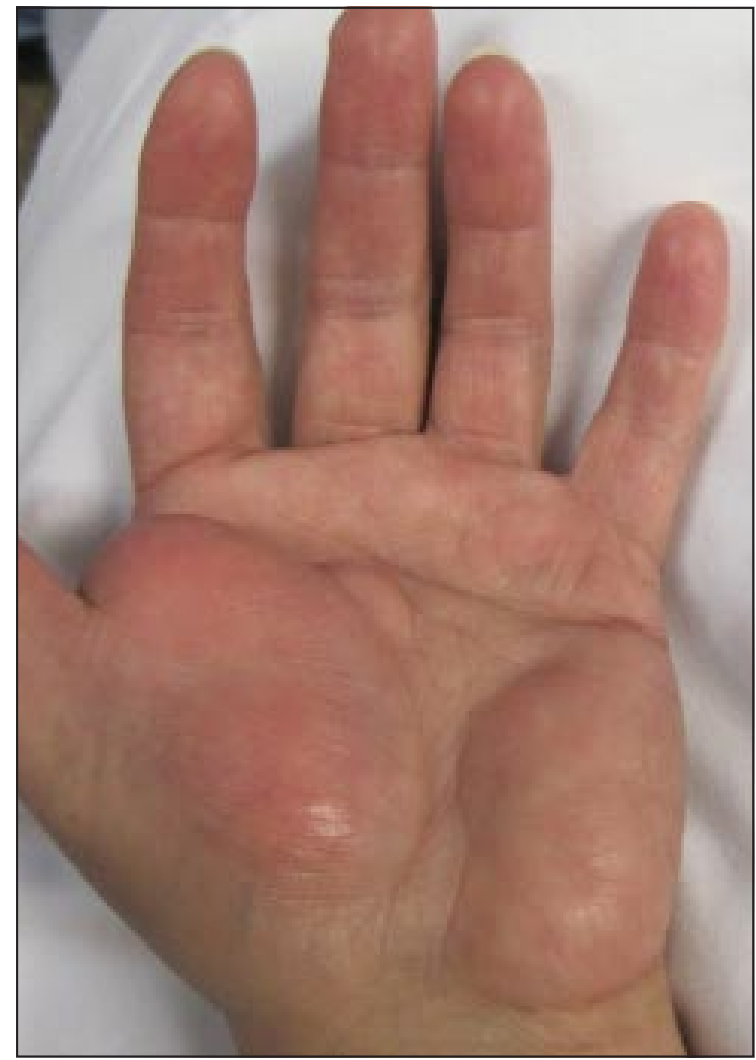

Figura 1. Imagen de la tumoración en la mano afecta de la paciente, previa a la cirugía.

da, bilobulada, de dos años de evolución, no dolorosa, con aumento progresivo de tamaño, que ocupaba la eminencia tenar e hipotenar. A la exploración física la tumoración era blanda, no adherida a planos profundos, con signo de la oleada positivo y sin cambios inflamatorios en la piel adyacente a la tumoración (Figura 1).

La paciente presentaba clínica compresiva del nervio mediano, como consecuencia de su

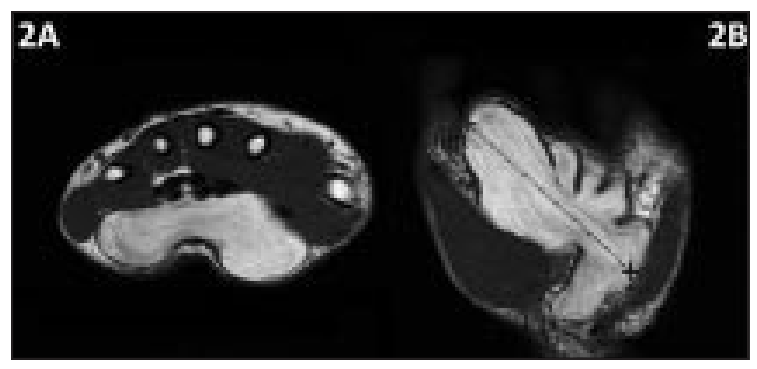

Figura 2. Imágenes de RM de la tumoración. 2A: corte axial; $2 B$ corte coronal en secuencia $T 2$.

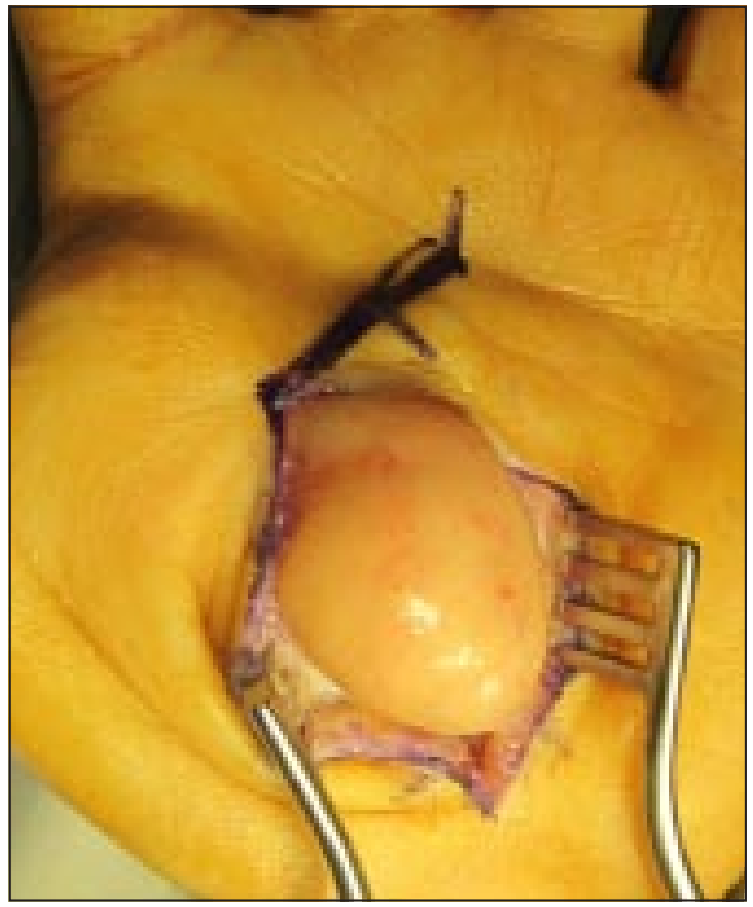

Figura 3. Imagen intraoperatoria del tumor tras incisión.

rápido crecimiento, con parestesias a nivel del segundo y tercer dedos.

Se realizó un estudio con RM, en el que se objetivó una tumoración de 7x6x5 cm localizada en espacio palmar profundo, desde la eminencia tenar hasta la hipotenar, que no invadía estructuras vasculonerviosas, ni músculotendinosas.

La tumoración era hiperintensa en las secuencias $\mathrm{T} 1$ e hipointensa en las secuencias T2, siendo hipointensa también en las secuencias con supresión grasa, lo que sugirió un origen graso de la lesión (Figura 2).

Dado el rápido crecimiento de la tumoración y su gran tamaño, se decidió, tras consentimiento informado de la paciente, intervenir quirúrgicamente.

Bajo anestesia regional y colocación de manguito de isquemia en el miembro superior, se realizó una incisión de unos 3-4 cm en la región palmar adyacente a la eminencia tenar. Tras apertura del ligamento anular del carpo, e identificación del paquete vasculonervioso, se procedió a la biopsia por escisión de la tumoración, que tenía un tamaño de $8 \mathrm{~cm}$ en su diámetro mayor. (Figuras 3-5). 


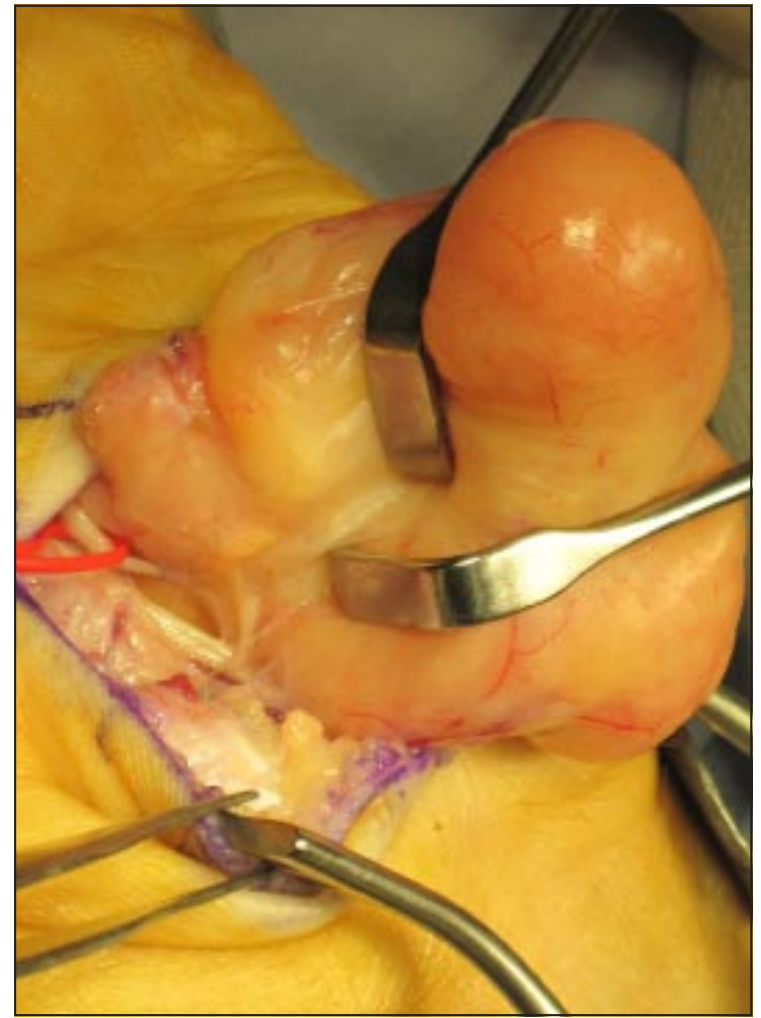

Figura 4. Disección del tumor, protegiendo al nervio mediano.

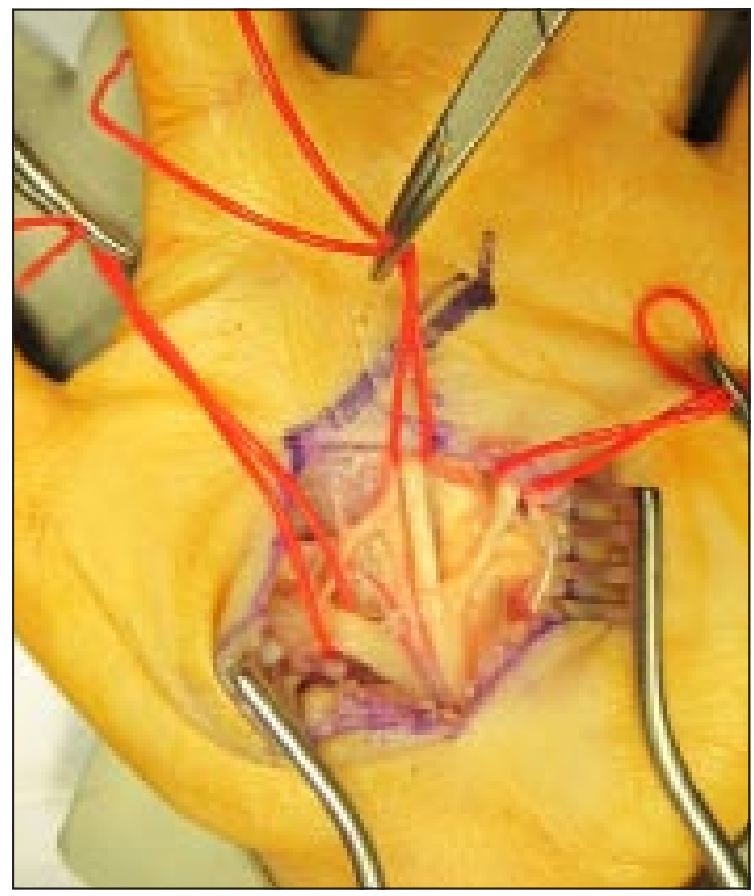

Figura 5. Anatomía de la región palmar de la mano, con identificación del nervio mediano y sus ramas, tras la escisión en bloque de la tumoración.

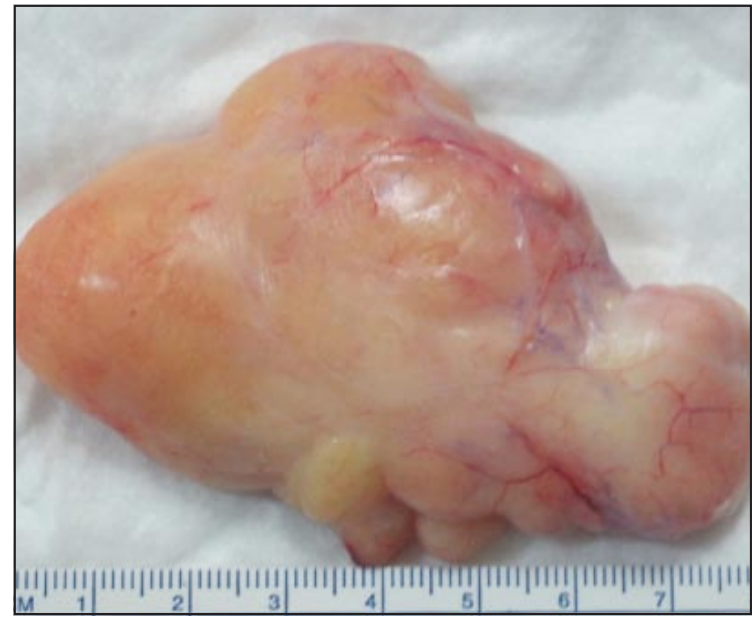

Figura 6. Imagen macroscópica del tumor, con $8 \mathrm{~cm}$. de diámetro mayor.

En el examen histopatológico posterior, se comprobó que, pese al gran tamaño de la lesión (Figura 6), se trataba de un angiolipoma, sin evidenciarse componentes de tejido nervioso, ni signos de malignización del mismo (Figura 7).

\section{RESULTADOS}

$\mathrm{Al}$ año de la lesión, la paciente se encuentra totalmente asintomática, sin signos de recidiva local, y con una correcta función de la mano.

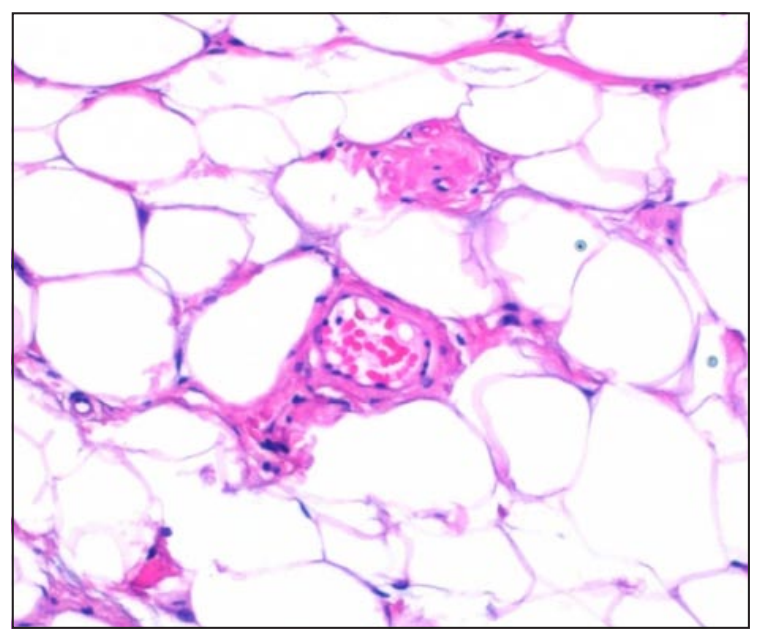

Figura 7. Anatomía patológica de la tumoración. Hematoxilina-eosina $\times 400$.

Revista Iberoamericana de Cirugía de la Mano 


\section{DISCUSIÓN}

Los lipomas son tumoraciones benignas muy frecuentes en el organismo pero que raramente se localizan en la mano. Generalmente se trata de tumoraciones de lento crecimiento y asintomáticas, a diferencia de lo que ocurría en esta paciente que presentaba clínica de compresión del nervio mediano, con parestesias asociadas.

Por otra parte, en otros casos descritos en los que los lipomas se asociaban con clínica compresiva, eran lesiones de crecimiento muy lento (hasta diez años) ${ }^{2}$, a diferencia del caso que presentamos, en el que el crecimiento fue mucho más rápido.

Dentro de los lipomas palmares, Oster et al. destacan la localización central de los lipomas, aunque en este caso se trató de una tumoración bilobulada que ocupaba las eminencias tenar e hipotenar así como la región central de la palma de la mano ${ }^{6}$.

Johnson et al. describen que los tumores mayores de $5 \mathrm{~cm}$ podrían ser considerados malignos, por su gran tamaño ${ }^{7}$, y se debe realizar el diagnóstico diferencial con el liposarcoma, por lo que se recomienda hacer un estudio anatomopatológico de la lesión, o una punción prequirúrgica de la misma ${ }^{8,9}$. En esta paciente, sin embargo, pese al gran tamaño de la lesión, no se evidenciaron signos de malignidad en el posterior estudio anatomopatológico de la pieza tumoral.

Son muy importantes los estudios de imagen preoperatorios, siendo de especial utilidad en los últimos años la $\mathrm{RM}^{10}$, que aporta información sobre las características de la tumoración, orientando así el diagnóstico de la misma. Además, nos permite la localización y delimitación exacta de la lesión (tamaño, relaciones con estructuras adyacentes), permitiendo así una buena preparación de la cirugía.

Es fundamental una buena planificación quirúrgica de la localización y tamaño de la incisión para poder extirpar adecuadamente la tumoración, y evitar la lesión de estructuras vasculonerviosas adyacentes ${ }^{11}$, siendo necesario, en ocasiones, la realización de doble abordaje.

$\mathrm{Si}$ se consigue una escisión completa de la tumoración, es rara la recidiva, quedando la paciente asintomática y con una función completa de la mano como ocurrió en el presente caso.

\section{CONCLUSIONES}

Los lipomas son tumores que rara vez se localizan en la mano. Si son de gran tamaño, pueden provocar una neuropatía compresiva del nervio mediano. Es importante para la planificación quirúrgica, el estudio previo con RM. Habitualmente la escisión completa de la tumoración, obtiene buenos resultados clínicos, siendo las recidivas infrecuentes. Un rápido crecimiento y un gran tamaño de la tumoración, deben hacer sospechar un posible carácter maligno, por lo que se debe optar por el tratamiento quirúrgico, y el posterior examen anatomopatológico permitirá el diagnostico diferencial con el liposarcoma de bajo grado.

\section{BIBLIOGRAFÍA}

1. Higgs PE, Young VL, Schuster R, Weeks PM. Giant lipomas of the hand and forearm. South Med J, 1993; 86: 887-90.

2. Fnini S, Hassoune J, Garche A, Rahmi M, Largab A. Lipome géant de la main: présentation d'un cas clinique et revue de la littérature. Chir Main, 2010; 29: 44-7.
3. Leffert RD. Lipomas of the upper extremity. J Bone Joint Surg Am, 1972; 54: 1262-6.

4. Booher RJ. Lipoblastic tumors of the hands and feet: Review of literature and report of thirtythree cases. J Bone Joint Surg Am, 1965; 47: 727-40.

5. Millán J, Serra JM, Morelli, A, Morelli E. Tumores de la mano, revisión de 250 casos. Rev Esp Cir Mano, 1985: 13: 25-42.

6. Oster LH, William FB, Curtis MS. Large lipomas in the deep palmar space. J Hand Surg Am, 1989; 14: 700-4.

7. Johnson CJ, Pynsent PB, Grimer RJ. Clinical features of soft tissue sarcomas. Ann Roy Coll Surg Eng, 2001; 83: 203-5. 
8. Mentzel T, Toennissen J, Rütten A, Schaller J. Palmar atypical lipomatous tumour with spindle cell features (well-differentiated spindle cell liposarcoma): a rare neoplasm arising in an unusual anatomical location. Virchows Arch, 2005; 446: 300-4.
9. Laurino L, Furlanetto A, Orvieto E, Del Tos AP. Well-differentiated liposarcoma (atypical lipomatous tumors). Semin Diagn Pathol, 2001; 18: 258-62.

10. Santos-Maraver MT, Collado Torres F, De la Torre Solís F, Rosón Méndez-Telles JJ. Lipomas de la mano. Cuatro nuevo casos. Revisión bibliográfica. Rev Ortop Traum, 1994; 38 IB: 297-301.

11. Babins DM, Lubahn JD. Palmar lipomas associated with compression of the median nerve. $\mathrm{J}$ Bone Joint Surg Am, 1994; 76: 1360-2. 